Rev. Adm. Saúde - Vol. 18, № 72, jul. - et. 2018

http://dx.doi.org/10.23973/ras.72.117

ARTIGO ORIGINAL

\title{
Monitoramento epidemiológico como instrumento de apoio à gestão de saúde: análise das notificações de leishmaniose visceral em Sobral, Ceará
}

Epidemiological monitoring as an instrument to support health management: analysis of reports of visceral leishmaniasis in Sobral, Ceará

\section{Camila Paiva Martins ${ }^{1}$, Maria Girlane Sousa Albuquerque Brandão', Milenna de Mesquita Braga1, Luiza Bruna Freire Sampaio1, Lívia Moreira Barros $^{2}$, Jéssica Costa Brito Pacheco ${ }^{3}$}

1. Acadêmica do curso de graduação em enfermagem da Universidade Estadual Vale do Acaraú - UVA, Sobral CE.

2. Enfermeira. Docente do curso de enfermagem da Universidade Estadual Vale do Acaraú UVA, Sobral CE.

3. Enfermeira especialista em terapia intensiva e cardiologia. Enfermeira Assistencial da Emergência Pediátrica do Hospital Regional Norte de Sobral, Sobral CE.

\section{RESUMO}

Objetivo: Conhecer o perfil epidemiológico da leishmaniose visceral e sua distribuição no município de Sobral CE, no período de 2007 a 2017. Material e métodos: Trata-se de estudo epidemiológico, descritivo, transversal, retrospectivo, com abordagem quantitativa, a partir de dados dos casos de leishmaniose visceral diagnosticados no período de 2007 a 2017 no município de Sobral CE notificados no Sistema de Informação de Agravos de Notificação (SINAN) do Ministério da Saúde. Resultados: Os dados do estudo mostram que no período foram notificados 874 casos de leishmaniose visceral no município de Sobral CE, sendo a maioria do sexo masculino $(63,5 \%)$, acometendo majoritariamente a raça parda $(90,6 \%)$ e apresentando maior prevalência na faixa etária entre 1 e 9 anos de idade (40,3\%). Identifica-se a associação entre maior incidência de leishmaniose visceral e piores condições 
de vida e baixa escolaridade, o que reforça indicações de que sua ocorrência está associada à situação de vulnerabilidade social na qual se encontra uma grande parte da população. Conclusão: Considerando que diversos fatores podem influenciar no processo saúde-doença, vê-se a necessidade da utilização de indicadores epidemiológicos, que reflitam o quadro real das condições de saúde da população. Nesse contexto, a conscientização das populações, além do apoio dos profissionais de saúde na criação de ações profiláticas, pode contribuir para o planejamento e a implantação de estratégias de controle da patologia de forma eficaz.

Palavras-chave: epidemiologia; leishmaniose visceral; saúde pública.

\section{ABSTRACT}

Objective: To know the epidemiological profile of visceral leishmaniasis and its distribution in the municipality of Sobral CE, from 2007 to 2017. Material and methods: This is an epidemiological, descriptive, cross-sectional, retrospective study with a quantitative approach based on data of the cases of visceral leishmaniasis diagnosed in the period 2007 to 2017 in the municipality of Sobral CE reported in the Ministry of Health's Notification of Injury Information System (SINAN). Results: The data from the study indicate that, in this period, 874 cases of visceral leishmaniasis in the city of Sobral CE, being the majority male (63,5\%), mainly affecting brown breed (90,6\%) and presenting a higher prevalence in the age range between 1 and 9 years of age (40,3\%). The association between a higher incidence of visceral leishmaniasis and poorer living conditions and low levels of education is identified, which reinforces indications that its occurrence is associated with the social vulnerability situation in which a large part of the population lives. Conclusion: Considering that several factors can influence the health-disease process, it is necessary to use epidemiological indicators, which reflect the real health conditions of the population. In this context, popular awareness, in addition to the support of health professionals in the creation of prophylactic actions, can contribute to the planning and implementation of effective pathology control strategies.

Keywords: epidemiology; visceral leishmaniasis; public health.

\section{INTRODUÇÃO}

As leishmanioses são enfermidades tropicais ocasionadas por protozoários do gênero Leishmania e disseminadas por mosquitos da família dos flebotomíneos. Atingem mais de 350 milhões de pessoas que vivem em áreas de risco distribuídas em 88 países, dos quais 72 são classificados como países 
em desenvolvimento. Acometem principalmente as pessoas mais pobres e com maior dificuldade de acesso aos serviços de saúde ${ }^{1}$.

A leishmaniose visceral (LV) destaca-se entre os diversos tipos de leishmanioses por tratar-se de uma doença infecciosa de caráter zoonótico que é capaz de acometer várias espécies de mamíferos, inclusive o homem, quando há o contato com o ciclo de transmissão do parasita. No Brasil, a Leishmania chagasi é o principal agente etiológico, sendo transmitida pelo inseto hematófago da espécie Lutzomya longipalpis e L. Cruzi $i^{2,3}$.

Apresenta-se clinicamente como uma enfermidade generalizada, de evolução crônica e sistêmica, podendo atingir $90 \%$ de letalidade se não tratada adequadamente ${ }^{4}$. No homem, a febre é a manifestação mais evidente, com característica irregular e de longa duração, seguida de hepatoesplenomegalia, atonia muscular, anemia, sinais de desnutrição proteicocalórica, dentre outras manifestações menos frequentes ${ }^{5}$.

A maior probabilidade de infecção ocorre em função de vulnerabilidades como precária infraestrutura sanitária, criação de animais que atuam como reservatórios do parasita, condições climáticas favoráveis à proliferação do vetor, cobertura vegetal com predomínio de árvores frutíferas, crescimento desordenado de áreas urbanas com acúmulo da matéria orgânica e negligência dos primeiros sintomas da doença ${ }^{6}$.

Segundo dados do Ministério da Saúde ${ }^{7}$, no ano de 2015, a região Nordeste do país apresentou o maior coeficiente de incidência da doença, com 3,2 casos por 100.000 habitantes, seguida da região Norte, com 2,7 casos.

Sabendo que a leishmaniose consiste em um grave problema de saúde pública, sendo um desafio tanto para os profissionais da saúde quanto para as autoridades competentes, é de extrema valência a promoção de estudos que determinem o padrão de eventualidade da LV e suas peculiaridades epidemiológicas, contribuindo assim para compreensão da dinâmica desse problema, bem como proporcionar a realização de ações de vigilância e gestão em saúde.

Deste modo, este estudo teve como objetivo descrever o perfil epidemiológico da leishmaniose visceral e sua distribuição espacial no município de Sobral no período de 2007 a 2017.

\section{MATERIAL E MÉTODOS}

Trata-se de um estudo epidemiológico, descritivo, transversal, retrospectivo, com abordagem quantitativa, a partir de dados dos casos leishmaniose visceral diagnosticados no período de 2007 a 2017 no município de Sobral, Ceará, e notificados no Sistema de Informação de Agravos de Notificação (SINAN) do Ministério da Saúde.

A epidemiologia descritiva examina como a incidência (casos novos) ou a prevalência (casos existentes) de uma doença, ou condição relacionada à 
saúde, varia de acordo com determinadas características dos indivíduos como sexo, idade, escolaridade e renda, entre outras, com fim de identificar grupos de risco para ações preventivas, e gerar hipóteses etiológicas para investigações futuras ${ }^{8}$.

A coleta de dados foi realizada nos meses de fevereiro e março de 2018, por meio de um formulário criado pelos autores, a partir de dados do SINAN, referente aos casos de leishmaniose visceral.

A população de estudo foi constituída pelos casos de leishmaniose visceral que ocorreram no município de Sobral no período de janeiro de 2007 a dezembro de 2017, estratificados pelas seguintes variáveis: zona de notificação, escolaridade, faixa etária, cor/raça, sexo e evolução clínica do caso.

Tendo em vista os aspectos operacionais da pesquisa, foi realizada uma análise estatística descritiva na íntegra, no intuito de descrever e sumarizar as variáveis dos casos notificados nesse município. Foram excluídos dados anteriores a 2007 e os dados de anos posteriores a 2017 por ainda não se apresentarem no SINAN. Por se tratar de uma pesquisa de dados secundários e não envolver diretamente seres humanos, este estudo não foi submetido à avaliação do comitê de ética em pesquisa da instituição.

Os dados coletados junto às bases oficiais foram, inicialmente, inseridos em planilhas criadas com o software MS-Excel, para facilitar a coleta dos dados, e, em seguida, transportados para o software Prism GraphPad 5.0, no qual foi realizada a análise estatística descritiva.

\section{RESULTADOS}

Os dados do estudo apontam que, no período compreendido entre 2007 a 2017, foram notificados 874 casos de leishmaniose visceral no município de Sobral. A Figura 1 ilustra a distribuição dos casos notificados no período em análise. Pode-se perceber que os anos de 2007 e 2010 tiveram os menores números de notificações em comparação aos demais anos, sendo o maior número de notificações de leishmaniose visceral em 2011 e 2014. 


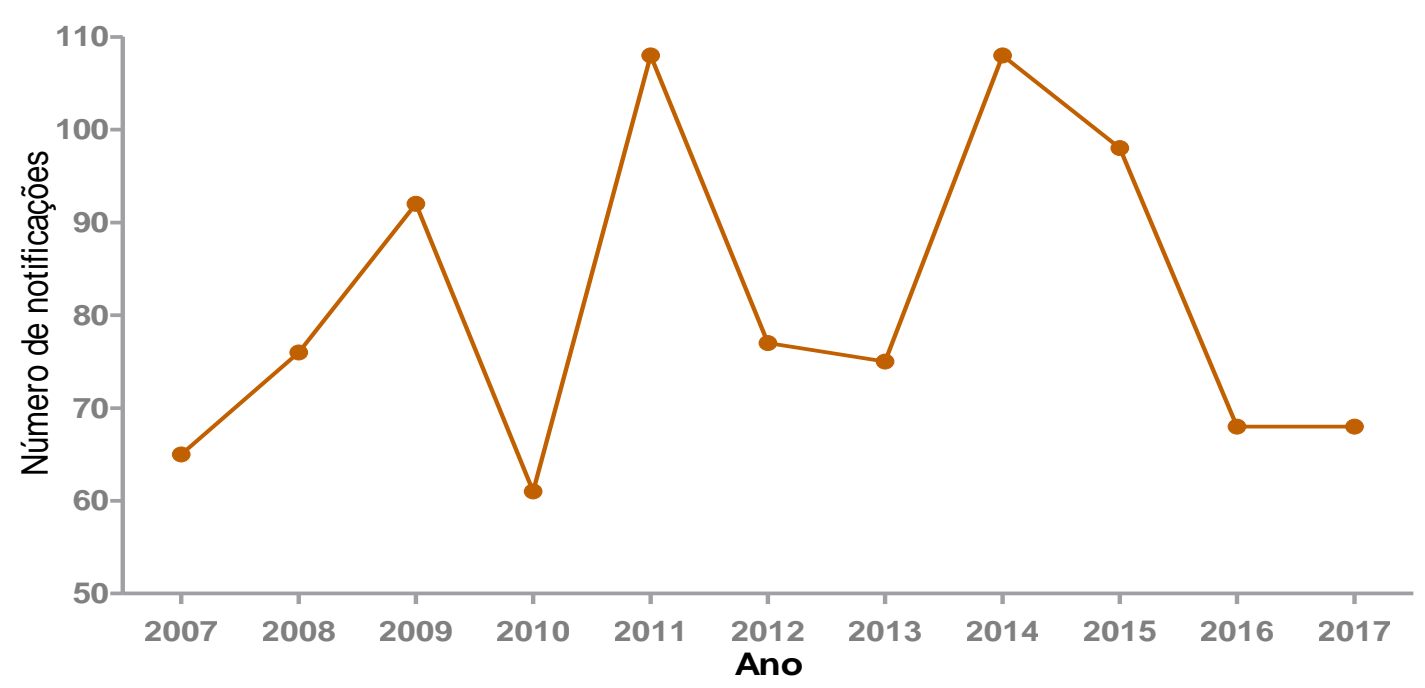

Figura 1. Evolução histórica dos casos de leishmaniose visceral, de 2007 a 2017, Sobral, Ceará.

É possível observar na Figura 1, que houve um aumento considerável no número de casos de leishmaniose visceral nos anos de 2011 e 2014. Em 2010, 2016 e 2017 o número de casos foi inferior.

Tabela 1. Distribuição dos casos de leishmaniose visceral notificadas por sexo, faixa etária, grau de escolaridade e cor/raça, 2007 a 2017, Sobral CE.

\begin{tabular}{lrr}
\hline SEXO & $\mathbf{N}$ & $\mathbf{\%}$ \\
\hline Masculino & 555 & 63,5 \\
\hline Feminino & 319 & 36,5 \\
\hline IDADE & $\mathbf{N}$ & $\%$ \\
\hline$<\mathbf{1}$ ano & 108 & 12,3 \\
\hline $\mathbf{1}$ a anos & 353 & 40,3 \\
\hline $\mathbf{1 0}$ a $\mathbf{1 9}$ anos & 90 & 10,2 \\
\hline $\mathbf{2 0}$ a $\mathbf{5 9}$ anos & 281 & 32,1 \\
\hline $\mathbf{6 0}$ a 79 anos & 38 & 4,3 \\
\hline >= 80 anos & 4 & 0,4 \\
\hline ESCOLARIDADE & $\mathbf{N}$ & $\mathbf{\%}$ \\
\hline Sem estudo & 9 & 1,0 \\
Ensino fundamental incompleto & 150 & 17,1 \\
Ensino fundamental incompleto & 9 & 1,0 \\
Ensino médio incompleto & 18 & 2,0 \\
Ensino médio completo & 17 & 1,9 \\
Ensino superior incompleto & 1 & 0,1 \\
Ensino superior completo & 1 & 0,1 \\
Ignorado & 256 & 29,2 \\
Não se aplica (*) & 413 & 47,2 \\
\hline COR/RAÇA & $\mathbf{N}$ & $\mathbf{\%}$ \\
\hline Branca & 43 & 4,9 \\
Parda & 792 & 90,6
\end{tabular}




\begin{tabular}{lll} 
Preta & 25 & 2,8 \\
Indígena & 02 & 0,2 \\
Ignorado & 12 & 1,3 \\
\hline
\end{tabular}

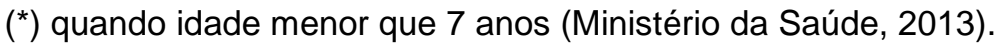

Fonte: SINAN, 2017.

Identificou-se que a faixa etária mais acometida foi a de 1 a 9 anos de idade $(40,3 \%)$. Em relação à escolaridade, a maior frequência foi em indivíduos com ensino fundamental incompleto $(17,1 \%)$ e a menor foi nos que possuíam ensino superior com $0,11 \%$. A cor/raça parda foi a mais acometida com a doença $(90,6 \%)$, e a menor foi a raça indígena $(0,2 \%)$.

Tabela 2. Distribuição dos casos de leishmaniose visceral por zona de notificação, critério de confirmação, tipo de entrada e evolução do caso, 2007 a 2017, Sobral CE.

\begin{tabular}{lrr}
\hline ZONA DE NOTIFICAÇÃO & $\mathbf{N}$ & $\%$ \\
\hline Urbana & 514 & 58,8 \\
\hline Rural & 334 & 38,2 \\
\hline Periurbana & 4 & 0,4 \\
\hline Ignorado & 22 & 2,5 \\
\hline CRITÉRIO DE CONFIRMAÇÃO & $\mathbf{N}$ & $\%$ \\
\hline Laboratorial & 823 & 94,1 \\
Clínico-epidemiológico & 51 & 5,8 \\
\hline TIPO DE ENTRADA & $\mathbf{N}$ & $\%$ \\
\hline Caso novo & 711 & 81,3 \\
Recidiva & 24 & 2,7 \\
Transferência & 120 & 13,7 \\
Ignorado & 19 & 2,1 \\
\hline EVOLUÇÃO & $\mathbf{N}$ & $\%$ \\
\hline Cura & 452 & 51,7 \\
Abandono & 3 & 0,3 \\
Óbito por LV & 51 & 5,8 \\
Óbito por outra causa & 12 & 1,3 \\
Transferência & 272 & 31,1 \\
Ignorado & 84 & 9,6 \\
\hline
\end{tabular}

Fonte: SINAN, 2017.

Em relação à zona de notificação dos casos de leishmaniose visceral, a maioria, $58,8 \%$, era da zona urbana. No que concerne ao critério de confirmação, $94,1 \%$ foram por meio de diagnóstico laboratorial, e 5,8\% deles, por meio da confirmação clínico-epidemiológica. Dentre os casos notificados, majoritariamente classificaram-se como caso novo (81,3\%). Sobre a evolução dos casos notificados de leishmaniose visceral, a maioria evoluiu para cura 
(51,7\%), entretanto, vale destacar a negatividade dos casos com evolução ignorada $(31,1 \%)$.

Na Figura 2, observando-se o número de óbitos por ano, se evidenciam dois picos nos anos de 2011 e 2014, anos nos quais foram identificados os maiores números de casos de leishmaniose no período estudado. Destaca-se também que o número de óbitos total (51 óbitos) representa um total de $5,8 \%$ dos casos notificados, mostrando que a leishmaniose é uma doença com alta letalidade atualmente na região.

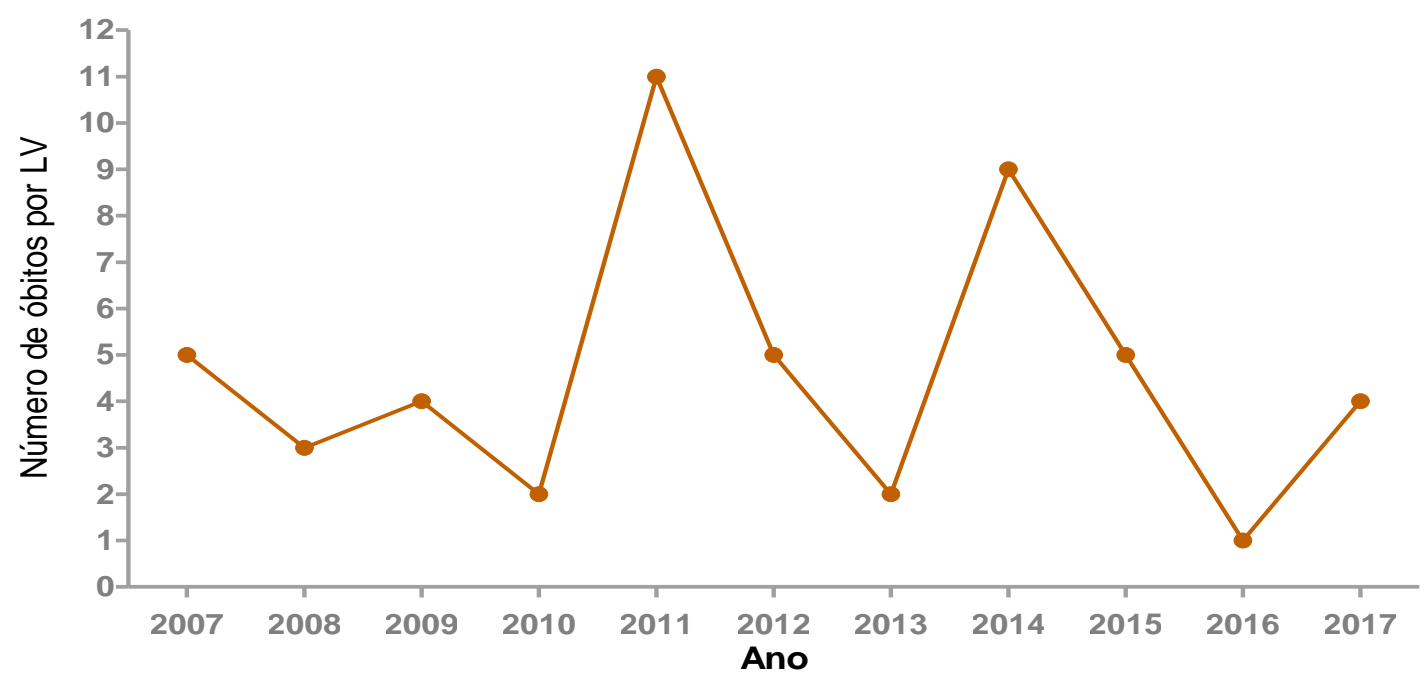

Figura 2. Distribuição da mortalidade por leishmaniose visceral, de 2007 a 2017, Sobral, Ceará.

\section{DISCUSSÃO}

A leishmaniose tem como agente etiológico protozoários do gênero Leishmania, sendo considerada uma zoonose que se manifesta através de úlceras ou por meio de acometimento sistêmico, com alta letalidade. A transmissão acontece quando fêmeas de insetos, infectadas após picar animais contaminados, transmitem a doença para animais silvestres ou domésticos, como cães, onde, a partir da contaminação eles passam a se tornar reservatórios dessa doença ${ }^{9}$. No caso dos animais domésticos, como eles estão mais próximos aos seres humanos, funcionam como a principal fonte de contaminação da leishmaniose.

A leishmaniose visceral é a forma mais grave da leishmaniose, devido às complicações frequentes e grandes taxas de mortalidade, caso não tratada. No ano de 2015, só no Brasil, foram notificados 3.289 casos da doença, representando $95,1 \%$ dos casos ocorridos nas Américas ${ }^{10}$. 
A leishmaniose visceral tem adquirido importância no contexto da saúde pública no Brasil devido a seu aumento significativo nos últimos anos. O estado do Ceará ainda é uma área endêmica para leishmaniose visceral. Em Sobral, um dos municípios da região noroeste do estado, que conta com uma área de $2.122,897 \mathrm{~km}^{2}$ e possui uma população estimada de 205.529 habitantes ${ }^{11}$, manifesta, historicamente, uma situação endêmica ${ }^{12}$ e tem mantido números elevados de casos a partir do ano de 2011, apresentando uma média de 93,4 casos entre 2011 e 2015 e alcançando uma taxa de mortalidade de 6,1\% relacionada à doença.

Antes prevalente na população rural, o maior número de casos da leishmaniose visceral na zona urbana $(58,8 \%)$ pode ser explicado pelas migrações intensas da população da zona rural, o que pode acarretar em ocupações desordenadas em áreas inadequadas, que provocam mudanças nos habitats das espécies envolvidas no ciclo de transmissão ${ }^{13}$. Ademais, de acordo com os dados do Censo Demográfico (2010), observou-se que $88 \%$ do total populacional de Sobral é representado por população urbana, sendo esta proporção superior as taxas médias estadual e nacional, que atingiram $86 \%$ e $85 \%$, respectivamente ${ }^{14}$.

Pesquisas demonstram que a leishmaniose visceral atinge principalmente a população que possui baixo nível socioeconômico, mais próximas ao vetor, se tornando suscetíveis à contaminação ${ }^{13,15,16}$. Dessa forma, saneamento básico e condições de vida precárias, auxiliam na exposição da população aos fatores de risco da doença. Os homens foram os mais acometidos pela doença $(63,5 \%)$, corroborando com uma pesquisa realizada em Roraima, em que $57,9 \%$ dos atingidos pela leishmaniose visceral eram do sexo masculino ${ }^{17}$.

Observa-se também que as crianças têm sido um grupo bastante vulnerável ao longo dos anos, apresentando uma taxa de 12,3\% em menores de um ano de idade e $40,3 \%$ na faixa etária entre um e nove anos, comparando-se a todos os casos apresentados no município. Pesquisa realizada em Recife PE, demonstrou maior letalidade de leishmaniose visceral em crianças de um a nove anos ${ }^{13}$. De acordo com o Ministério da Saúde, esses valores estão relacionados a pouca maturidade imunológica da criança, onde as doenças são mais frequentes nessa fase da vida, podendo estar associada com a desnutrição, considerada fator de risco em países em desenvolvimento, como o Brasil $^{9}$.

Com relação à cor/raça, identifica-se um número expressivo de casos na população parda $(90,6 \%)$. Todavia, vale destacar que as informações relativas à cor/raça da população de Sobral sinalizam que a maior parcela dos habitantes declarou ser parda, reunindo mais de 118 mil pessoas, de acordo com o IBGE (2010). Este contingente representava $63 \%$ do total populacional, característica semelhante ao perfil declarado no estado do Ceará (equivalente a $62 \%$ ). Em seguida, os habitantes do município eram representados por brancos, o que envolvia 55.632 indivíduos e $29 \%$ do total ${ }^{14}$.

Não há estudos que associem o risco de adquirir a leishmaniose visceral pela etnia, entretanto, pode-se conferir a esses dados a ocorrência de riscos relacionados às condições socioeconômicas dessa população, como pouca 
renda familiar, baixa escolaridade ( $17,1 \%$ - ensino fundamental incompleto) e pouco acesso às informações relacionadas à doença. Um estudo realizado no estado de Minas Gerais identificou que $67,2 \%$ da população acometida por essa patologia era da cor/raça parda ou preta ${ }^{18}$.

Assim, verifica-se a correlação entre maiores incidências de leishmaniose visceral e piores condições de vida e baixa escolaridade, reforçando que sua ocorrência está associada à situação de vulnerabilidade social na qual se encontra grande parte da população ${ }^{15,16}$.

Quanto à evolução clínica dos casos, 51,7\% evoluíram para cura. Para as situações abandono de tratamento e transferência, registraram-se $0,3 \%$ e $31,1 \%$, respectivamente. É relevante considerar que uma parcela dos indivíduos com LV foi a óbito, mostrando uma letalidade crescente e alta $(5,8 \%)$, quando comparado ao estimado pelo Ministério da Saúde ${ }^{19}$. Segundo dados deste órgão, a letalidade por LV aumentou de 3,4\% em 1994 para 5,7\% em 2009, o que representou incremento de $67,6 \%{ }^{19}$. Nesse contexto, salientase a importância das medidas de controle e prevenção, bem como de diagnóstico e tratamento precoce.

As medidas de controle da doença estão associadas ao vetor. Para evitar a transmissão, podem ser utilizadas algumas medidas simples de proteção, como uso de mosquiteiro, colocação de telas em portas e janelas além uso de repelentes nos locais onde o vetor pode ser encontrado ${ }^{9}$.

Com relação ao ambiente, medidas simples como a limpeza de quintais, terrenos e eliminação de resíduos que podem propiciar a formação de criadouros, contribuem para evitar a proliferação do vetor. Ademais, o controle da doença através da busca ativa de cães sintomáticos e em situação de rua é essencial, pois são fontes disseminadoras da doença.

A educação dos sujeitos envolvidos no contexto econômico e social mais vulnerável à leishmaniose atua como ferramenta para a promoção da saúde, pois apropriação do ciclo de contaminação da doença auxilia na capacitação dessa população, criando estratégias para prevenção da doença, pautadas na articulação entre os serviços de saúde, além da criação de meios de higiene e nutrição saudável.

Vale destacar a instituição da lei federal 12.604/12, que cria a Semana Nacional de Controle e Combate à Leishmaniose, que tem entre seus objetivos estimular ações educativas e preventivas, bem como promover debates e outros eventos sobre as políticas públicas de vigilância e controle da leishmaniose, além de apoiar as atividades de prevenção e combate, organizadas e desenvolvidas pela sociedade civil $^{20}$.

A implantação de estratégias como essas, contando com o apoio institucional e participação de profissionais na assistência e vigilância em saúde, em conjunto com a população, corroboram para o êxito na diminuição do número de casos da doença ${ }^{21}$. 
Os indicadores epidemiológicos refletem o quadro real das condições de saúde de uma população. Sua utilização pode orientar os gestores de saúde no planejamento e controle das atividades locais. As informações providas pelos indicadores fornecem o embasamento necessário ao planejamento, à execução e à avaliação das ações realizadas, na medida em que propiciam o conhecimento sobre aspectos relevantes da população, reduzem o grau de incerteza sobre sua situação de saúde e apoiam a busca de possíveis soluções e providências ${ }^{22}$.

\section{CONCLUSÃO}

Foi possível evidenciar neste estudo que na série histórica de 2007 a 2017 foram notificados 874 casos de leishmaniose visceral, predominante em indivíduos da cor/raça parda, de baixa escolaridade e na faixa etária de um a nove anos de idade, sendo 2011 e 2014 os anos que apresentaram os maiores números de notificações.

Considerando que diversos fatores podem influenciar no processo saúdedoença, vê-se a necessidade da utilização de indicadores epidemiológicos, que reflitam o quadro real das condições de saúde da população. A compreensão do perfil epidemiológico da leishmaniose é de relevância para a constituição de estratégias, especialmente para os grupos mais acometidos, pois sua utilização pode orientar os gestores de saúde no planejamento e controle das atividades locais.

Para tanto, é importante que profissionais da saúde e gestores estejam envolvidos diretamente no desenvolvimento de estratégias, visando à melhoria da qualidade do serviço prestado.

A interpretação dos resultados deste estudo deve levar em consideração as limitações decorrentes da utilização de dados do SINAN. Esses dados, apesar de terem sua importância definida, estão sujeitos a subnotificações, além de erros eventuais por problemas de completude e consistência, que se traduzem em problemas de quantidade e qualidade da informação.

Vale ainda realçar os inúmeros valores ignorados ou brancos nas variáveis analisadas neste estudo, o que pode depreciar a qualidade dos registros dos sistemas de informação, de forma a dificultar a realização de estudos epidemiológicos precisos com base no SINAN. Assim, a adequada notificação pelos profissionais de saúde é fundamental para o controle da doença, de modo que possibilite a investigação e o correto acompanhamento dos casos.

Entretanto, a abordagem desses dados é de relevância para despertar o desenvolvimento de ações de vigilância em saúde e a tomada de medidas preventivas, uma vez que o estado do Ceará constitui-se como uma área endêmica, não podendo, então, ser negligenciada, pois é uma doença que vem modificando suas características ao longo do tempo, ganhando espaço na zona urbana, local onde vive hoje grande parte da população. 
Nesse contexto, a conscientização e capacitação das populações, além do apoio dos profissionais de saúde na correta notificação e ações profiláticas, podem contribuir para a implantação de estratégias de controle eficazes, revertendo a realidade preocupante na qual se encontra o município.

\section{REFERÊNCIAS}

1. Batista FMA, Machado FFOA, Silva JMO, Barja, PR, Mittmann J, Simioni AR. Leishmaniose: perfil epidemiológico dos casos notificados no estado do Piauí entre 2007 e 2011. Rev Univap [Internet]. 2014 [citado 2018 Fev 26]; 20(35): 02-13. Disponível em:

https://revista.univap.br/index.php/revistaunivap/article/view/180

2. Filippis T, Neves, DP. Parasitologia Básica - 2ª Ed. Atheneu, 2010.

3. Barbosa IR, Neto RDS, Souza PP, Silva RA, Lima SR, Cruz IDD, Costa ICC. Aspectos da coinfecção leishmaniose visceral e HIV no nordeste do Brasil. Rev Baiana de Saúde Pública [Internet]. 2013 [citado 2018 Fev 26]; 37(3): 672-687. Disponível em:

http://bases.bireme.br/cgibin/wxislind.exe/iah/online/?IsisScript=iah/iah.xis\&src= google\&base $=$ LILACS\&lang=p\&nextAction=Ink\&exprSearch=728982\&indexSea $\underline{\mathrm{rch}=\mathrm{ID}}$

4. Oliveira JM, Fernandes AC, Dorval MEC, Alves TP, Fernandes TD, Oshiro ET, et al. Mortalidade por leishmaniose visceral: aspectos clínicos e laboratoriais. Revista da Sociedade Brasileira de Medicina Tropical [Internet]. 2010 [citado 2018 Fev 26]; 43(2):188-93. Disponível em: http://www.scielo.br/pdf/rsbmt/v43n2/16.pdf

5. Brasil. Ministério da Saúde. Secretaria de Vigilância em Saúde. Guia de Vigilância em Saúde. Brasília: Editora do Ministério da Saúde. [Internet]. 2017. Disponível em:

http://portalarquivos.saude.gov.br/images/pdf/2017/outubro/06/Volume-Unico2017.pdf

6. Silva LB, Aquino DMC, Bezerra JMT, Melo MN, Leonardo FS, Silva ASG, Pinheiro VCS. Fatores associados à leishmaniose visceral na área endêmica de Codó, estado do Maranhão, Brasil. Revista de Epidemiologia e Controle de Infecção [Internet] 2016 [citado 2018 Fev 29]; 6(2): 74-80. Disponível em: https://online.unisc.br/seer/index.php/epidemiologia/article/view/6419/5066

7. Brasil. Ministério da Saúde. Secretaria de Vigilância em Saúde. Coeficiente de incidência de leishmaniose visceral por 100.000 habitantes. Brasil, Grandes Regiões e Unidades Federadas. 1990 a 2015. [Internet]. Brasília, 2017.

Disponível em:

http://portalarquivos.saude.gov.br/images/pdf/2016/novembro/08/LVCoeficiente\%20de\%20Incidncia.pdf 
8. Trujillo AM. Epidemiologia: história, tipos e métodos. Revista Simbiótica [Internet]. 2016 [citado 2018 Jun 18]; 3(1):1-27. Disponível em:

http://periodicos.ufes.br/simbiotica/article/view/14624

9. Brasil. Ministério da Saúde, Secretaria de Vigilância em Saúde,

Departamento de Vigilância Epidemiológica. Manual de vigilância e controle da leishmaniose visceral [Internet]. Brasília (DF): Ministério da Saúde; 2014. (Série A. Normas e manuais técnicos). Disponível em:

http://bvsms.saude.gov.br/bvs/publicacoes/manual vigilancia controle leishma niose visceral 1edicao.pdf

10. Organização Mundial da Saúde. Situação epidemiológica da Leishmaniose [Internet]. 2015. Disponível em: http://www.who.int/leishmaniasis/burden/en/

11. IBGE. População estimada. Diretoria de Pesquisas, Coordenação de População e Indicadores Sociais, Estimativas da população residente com data de referência $1^{\circ}$ de julho de 2017 [Internet]. 2017. Disponível em:

https://cidades.ibge.gov.br/v4/brasil/ce/sobral/panorama

12. Cavalcante IJM, Vale MR. Aspectos epidemiológicos da leishmaniose visceral (calazar) no Ceará no período de 2007 a 2011. Rev. bras. epidemiol [Internet]. 2014 [citado 2018 Jun 18]; 17(4): 911-924. Disponível em: http://repositorio.ufc.br/bitstream/riufc/9843/1/2014 art ijmcavalcante.pdf

13. Maia CS, Pimentel DS, Santana MA, Oliveira GM, Pedrosa NA, Nascimento $L A$, et al. espacial da leishmaniose visceral americana no município de Petrolina, Pernambuco, Brasil [Internet]. Hygeia, 2014 [citado 2018 Mar 01]; 10(18):167-176. Disponível em:

http://www.seer.ufu.br/index.php/hygeia/article/viewFile/24027/14857

14. Prefeitura Municipal de Sobral. Diagnóstico Integrado [Internet]. Sobral; 2016 [citado 2018 Jul 06]. Disponível em: http://sobraldefuturo.com.br/wpcontent/uploads/2017/06/Diagnostico-Integrado 2106-1.pdf

15. Toledo CRS, Almeida AS, Chaves SAM, Sabroza PC, Toledo LM, Caldas JP. Vulnerabilidade à transmissão da leishmaniose visceral Humana em área urbana brasileira. Rev Saúde Pública [Internet]. 2017 [citado 2018 Mar 01]; 51(49):1-11. Disponível em: http://www.scielo.br/pdf/rsp/v51/pt 0034-8910-rsp$\underline{\text { S1518-87872017051006532.pdf }}$

16. Silva AR, Tauil PL, Cavalcante MNS, Medeiros MN, Pires BN, Gonçalvez EGR. Situação epidemiológica da leishmaniose visceral, na llha de São Luís, Estado do Maranhão. Rev Soc Bras Med Trop [Internet]. 2008 [citado 2018 Mar 02]; 41(4):358-64. Disponível em:

http://www.scielo.br/pdf/rsbmt/v41n4/a07v41n4.pdf

17. Evangelista LSM, Sibajev A. Perfil epidemiológico da leishmaniose visceral no estado de Roraima. BEPA, Bol. Epidemiol [Internet]. 2012 [citado 2018 Mar 02]; 9(102): 30-35. Disponível em:

http://periodicos.ses.sp.bvs.br/scielo.php?script=sci arttext\&pid=S1806$42722012000600004 \& \operatorname{lng}=$ pt. 
18. Oliveira EN, Pimenta AM. Perfil epidemiológico das pessoas portadoras de leishmaniose visceral no município de Paracatu - MG no período de 2007 a 2010. Rev Min Enferm [Internet]. 2014 [citado 2018 Mar 02]; 18(2): 365-370. Disponível em: http://www.reme.org.br/artigo/detalhes/933

19. Barata RA, Silva JCF, Silva JC, Almeida SN, Teixeira LAS, Dias ES.

Controle da leishmaniose visceral no município de Porteirinha, Estado de Minas Gerais, no período de 1998 a 2003 [Internet]. Rev. Soc. Bras. Med. Trop. 2011 [citado 2018 Jul 07]; 44(3): 386-88. Disponível em: http://www.scielo.br/pdf/rsbmt/v44n3/v44n 3a25.pdf

20. Alvar J, Vélez DI, Bern C, Herrero M, Desjeux P, Cano J,et al. Leishmaniasis Control Team. Leishmaniasis Worldwide and Global Estimates of Its Incidence.PloS One [Internet]. 2012. [citado 2018 Jul 07];7(5): 35671 Disponível em: https://www.ncbi.nlm.nih.gov/pubmed/22693548

21. Lima KWS, Antunes JLF, Silva, ZP. Percepção dos gestores sobre o uso de indicadores nos serviços de saúde. Saúde Soc [Internet]. 2015 [citado 2018 Mar 03]; 24(1):61-71. Disponível em: http://www.scielo.br/pdf/sausoc/v24n1/0104-1290-sausoc-24-1-0061.pdf

22. Oliveira LS, Neto RVD, Braga PET. Perfil epidemiológico dos casos de leishmaniose visceral em Sobral, Ceará no período de 2001 a 2010. Sanare, Sobral, V.12, n.1, p. 13-19, jan./jun. - 2013. [citado 2018 Jul 06] Disponível em: https://sanare.emnuvens.com.br/sanare/article/view/323/258

Recebido: 17 de junho de 2018. Publicado: 16 de julho de 2018

Correspondência: Maria Girlane Sousa Albuquerque Brandão. E-mail: girlane.albuquerque@yahoo.com.br

Conflito de interesses: os autores declararam não haver conflito de interesses.

(C) This is an Open Access article distributed under the terms of the Creative Commons Attribution License, which permits unrestricted use, distribution, and reproduction in any medium, provided the original work is properly cited 